\title{
BMJ open Attitudes and beliefs about hand hygiene among paediatric residents: a qualitative study
}

\author{
Devika Dixit, ${ }^{1}$ Reidar Hagtvedt, ${ }^{2}$ Trish Reay, ${ }^{3}$ Mark Ballermann, ${ }^{4}$ Sarah Forgie ${ }^{1}$
}

To cite: Dixit $D$, Hagtvedt $R$, Reay $\mathrm{T}$, et al. Attitudes and beliefs about hand hygiene among paediatric residents: a qualitative study. BMJ Open 2012;2: 002188.

doi:10.1136/bmjopen-2012002188

- Prepublication history and additional material for this paper are available online. To view these files please visit the journal online (http://dx.doi.org/10.1136/ bmjopen-2012-002188).

Received 8 October 2012 Revised 3 November 2012 Accepted 15 November 2012

This final article is available for use under the terms of the Creative Commons Attribution Non-Commercial 2.0 Licence; see http://bmjopen.bmj.com

\begin{abstract}
${ }^{1}$ Department of Paediatrics, Faculty of Medicine and Dentistry, University of Alberta, Edmonton, Canada ${ }^{2}$ Department of Accounting, Operations and Information Systems Alberta School of Business, University of Alberta, Edmonton, Canada ${ }^{3}$ Department of Strategic Management and Organization Alberta School of Business, University of Alberta, Edmonton, Canada ${ }^{4}$ Clinical Informatics North Alberta Health Services, Edmonton, Canada
\end{abstract}

Correspondence to Sarah Forgie, sarah.forgie@ albertahealthservices.ca

\section{ABSTRACT}

Background: Despite the knowledge that contaminated hands play an important role in the transmission of healthcare-associated pathogens, and that hand hygiene $(\mathrm{HH})$ reduces the transmission of these organisms, healthcare worker's adherence with $\mathrm{HH}$ is poor.

Objective: To understand the common beliefs and attitudes held by paediatric residents about $\mathrm{HH}$.

Design: Qualitative study design.

Setting: Tertiary care paediatric hospital in Edmonton, Canada.

Methods: Semistructured interviews were conducted and themes were identified from interviews.

Participants: 22 paediatric residents.

Results: Paediatric residents self-reported their $\mathrm{HH}$ adherence at $70-99 \%$ and perceived hospital-wide adherence at $45-80 \%$. Four major themes were identified during interviews including the importance of role modelling, balancing time spent on $\mathrm{HH}$ with other competing factors, self-protection as a driving factor for $\mathrm{HH}$ and cues as an important part of habit that stimulate $\mathrm{HH}$.

Conclusions: Staff physicians were viewed as integral to initiating group $\mathrm{HH}$ events, but at times, the first person in the room acted as a role model for the rest of the group. In certain instances, such as a cardiac arrest, decreased adherence with $\mathrm{HH}$ was viewed as acceptable. Residents engaged in $\mathrm{HH}$ to protect their own health. Residents relied on personal cues, which they integrated into their own $\mathrm{HH}$ habit. Future $\mathrm{HH}$ adherence strategies should ensure that the physician training environments permit the formation of good attitudes and habits towards $\mathrm{HH}$. There are no additional data available.

\section{INTRODUCTION AND BACKGROUND}

Contaminated hands play an important role in the transmission of healthcare-associated pathogens, and hand hygiene $(\mathrm{HH})$ reduces the transmission of these organisms. ${ }^{1-4}$ In the paediatric population, viral infections contribute significantly to nosocomial infections. ${ }^{5}$ At the Stollery Children's Hospital in Edmonton, Canada, over 1000 children are admitted with viral respiratory tract infections each year and $3-4 \%$ of children admitted for other reasons acquire viral respiratory infections while in

\section{ARTICLE SUMMARY}

Article focus

- The attitudes and beliefs about hand hygiene $(\mathrm{HH})$ among paediatric residents.

Key messages

- Our findings showed that paediatric residents' compliance with $\mathrm{HH}$ is influenced by role modelling, balancing $\mathrm{HH}$ with other competing factors, the drive for self-protection and personal cues.

Strengths and limitations of this study

- The strengths of this study include our sample, which originates in a large referral teaching hospital where $\mathrm{HH}$ compliance is quantitatively similar to that reported in the literature $(50 \%)$. This study focuses exclusively on paediatric resident physicians who are at a stage in their training where educational interventions may set the stage for lifelong habits. Selection bias was reduced since all paediatric residents were interviewed. The interview format of the study may have led to recall bias. Further studies are required to examine the relationship of these themes toHH compliance.

hospital. (Paediatric Respiratory Viral Illness Surveillance Summary Report. Stollery Children's Hospital. 1 November 2011-30 April 30 2012.)

On paediatric inpatient wards, viruses are most commonly transmitted from patient to patient and healthcare worker to patient by direct contact (and less commonly by respiratory droplets). ${ }^{6}$ Therefore, $\mathrm{HH}$ is an important measure to reduce the transmission of viruses between healthcare workers and patients. However, $\mathrm{HH}$ does not always occur. In the 2011 quarterly review of $\mathrm{HH}$ at our facility, overall $\mathrm{HH}$ average was observed to be $43.6 \%$ with the highest compliance $(78.5 \%)$ in the neonatal intensive care unit and the lowest compliance $(24.1 \%)$ on the paediatric cardiac inpatient ward (Alberta Health Services. Hand Hygiene Review. Summer 2011). There are multiple reasons 
described in the literature for low compliance with $\mathrm{HH}$ including lack of availability of $\mathrm{HH}$ material, time and workload issues, knowledge and education as well as staff attitudes regarding $\mathrm{HH}^{-7-9}$ Recently, interventions including strong leadership, increase in the availability of alcohol-based hand sanitisers and staff monitoring has led to increased $\mathrm{HH}$ compliance in the paediatric setting. ${ }^{10}$

We believe that a better understanding of the $\mathrm{HH}$ attitudes and beliefs among resident physicians may play a role in improving compliance with HH. Residents, or physicians in training, are in a unique position having recently finished a medical degree, but are still not at the level of an independently practicing physician. They are learners and teachers, and their attitudes and beliefs about infection prevention at this stage of training set the groundwork for their future as practicing physicians.

\section{THE STUDY}

\section{Aim/objective}

To understand the common beliefs and attitudes held by paediatric residents with regard to the adherence, or lack-thereof, with $\mathrm{HH}$ measures. In our study $\mathrm{HH}$ includes both hand washing using soap and water as well as the use of alcohol-based hand sanitisers.

\section{Methods}

A qualitative study design was employed to develop common themes regarding resident attitudes and beliefs about $\mathrm{HH}$.

\section{Sample}

This study was conducted at the Stollery Children's Hospital in Edmonton, Canada. The Stollery is a 135-bed tertiary care facility with $\mathrm{HH}$ facilities at each patient room and in the hallways. It is a 'hospital within a hospital' where the children's hospital is found within the adult hospital. Since 2003, all staff, including residents, receive infection control training on an annual basis. General paediatric residents in years 1 (initial) to 4 (final) of their training were interviewed during this study. Residents were recruited via email and participation in the study was voluntary and no incentives were given for participation. The paediatric residents were chosen as a sample because they are physicians in training which make them an interesting, convenient sample to study.

\section{Data collection}

Semistructured interviews with 22 paediatric residents were conducted for $45-60 \mathrm{~min}$ by the first author DD. An interview guide with open questions based on four major groupings (cues, interpersonal dynamics, context and models for $\mathrm{HH}$ adherence) was used. Rigour in data collection was maintained by using the interview guide as a framework for the interviews with similar wording and order of questions for all interviewees.
Interviews were digitally recorded and transcribed by a single transcriptionist and verified for accuracy by the research team. The transcribed interviews and audio files were kept in a secure location. The interview guide is attached in the online supplementary appendix. Data saturation was achieved after the 22 residents were interviewed and similar themes were identified from these interviews.

\section{Ethics}

Ethics approval for research was obtained through the Human Research Ethics Board at the University of Alberta in Edmonton, Canada. Written, informed consent was obtained from all study participants. All transcribed interviews were coded numerically to preserve anonymity during analysis.

\section{Data analysis}

Following qualitative interpretive methods, transcribed interviews were reviewed by the five authors and coded based on a coding system developed through iterative discussions among researchers. ${ }^{11}$ All interviews were read by the first author DD and four or five were read by each of the remaining researchers. Researchers coded interviews independently, and these codes were reviewed by the research team to achieve inter-rater consistency. Further codes were developed during group discussions based on emerging common themes. Interviews were then re-read and coded using the following categories: habit, characteristic of the setting, rules and regulations, breakdown, communication, ease of use $(\mathrm{HH}$ materials), patient factors and resident factors. The themes were then further refined and used in the next (and final) round of analyses. These final themes were importance of role models, trade-offs for when HH may be neglected because of other factors, self-protection and back up/recovery in near-miss situations. Final themes were ultimately refined to clarify intended meanings of themes including importance of role models, balancing competing priorities, self-protection and cues to initiate $\mathrm{HH}$ habit. We maintained rigour and validated the final identified themes by describing them to and gaining confirmation from our study participants to ensure accuracy of intended meaning. ${ }^{12}$ See figure 1 for theme development.

\section{RESULTS}

Twenty-two paediatric residents were interviewed. The residents varied in age from 24 to 40 and residents were predominately female $82 \%(18 / 22)$.

Four major themes were identified from the interviews including the importance of role modelling, balancing $\mathrm{HH}$ with other competing factors which may cause $\mathrm{HH}$ to be neglected, self-protection as a driving factor for $\mathrm{HH}$ and cues as an important part of habit that stimulate $\mathrm{HH}$. 


\section{Initial categories/themes:}

-Cues

-Interpersonal dynamics

-Context

-Models for hand-hygiene adherence

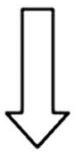

\section{Coding:}

-Habit

-Characteristic of the setting

-Rules and regulations

-Breakdown

-Communication

-Ease of use

-Patient factors

-Resident factors

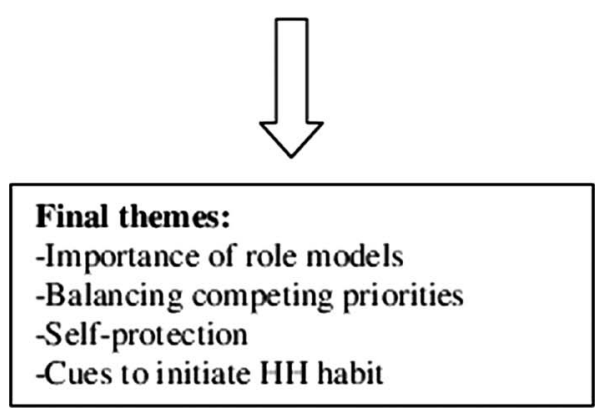

Figure 1 Theme development.

\section{Role modelling}

Role modelling was described as an important determinant of $\mathrm{HH}$, and was sometimes viewed in a hierarchical way. Individuals in leadership positions were viewed as responsible for modelling good $\mathrm{HH}$ behaviour. However, role modelling of $\mathrm{HH}$ behaviours could also be nonhierarchical, where, for example, the first person going into a patient room, or the medical student could initiate $\mathrm{HH}$ behaviours thereby acting as a cue and stimulus for others on the team.

\section{Residents as role models}

Senior residents perceived themselves as $\mathrm{HH}$ role models because $\mathrm{HH}$ is part of professional standards they strive to maintain as physicians:

...I know it $[\mathrm{HH}]$ does reduce the spread of infection. I think it also requires you to act as a role model for others that you are working with like medical students or any allied health care professionals. And also, like anyone in the health care profession, if you see someone not washing their hands and you think it is inappropriate, you have the responsibility to say something. Interview 4
Attending physicians acting as role models for $\mathrm{HH}$

Others reported that since as residents they are still learning, that they are greatly influenced by the $\mathrm{HH}$ performed by their staff physician and that this directly affects their own adherence to $\mathrm{HH}$.

The staff does [set the tone] and the residents do, but I think the staff have the biggest impact on how much hygiene you do. Interview 5

\section{Non-hierarchical role models}

The non-hierarchical aspect of $\mathrm{HH}$ where anyone has the ability to promote $\mathrm{HH}$ is exemplified in the quotes below:

The first person who approaches the room [is important] too. So, whoever is the first to start the chain almost, it's almost like a chain, so people will be cued by that. It doesn't necessarily have to be a specific person, but oftentimes if it is forgotten, then I think oftentimes any of the residents or staff will remind the team. Interview 6

The medical students quite often remind me in a demonstrative kind of way, like they are all stopping and washing their hands, so it kind of reminds me that if they are all doing it maybe I should do it too. Interview 7

You always take the lead of the person who has gone in in-front of you. So, if somebody else is doing it, you always follow. I do find the converse is true, so as a resident, if a staff has gone in without washing their hands, gowning or gloving, you just follow suit. Interview 15

\section{Negative role models}

At times, attending physicians could set a negative example and make it difficult for residents to adhere with good $\mathrm{HH}$ practices:

...by the time I had walked into the room [after completing $\mathrm{HH}]$, the staff physician had already assessed the patient and actually walked out... at that point I almost felt like maybe I shouldn't have done that and just ran into the room, seen the patient, and then left. Interview 8

\section{Balancing competing priorities}

At times lack of adherence with $\mathrm{HH}$ was viewed as acceptable. For example, residents treating acutely emergent patients described scenarios where they considered strictly adhering to $\mathrm{HH}$ as being difficult.

If you're going in to do CPR, then you're not going to stop and wash your hands. Interview 2

I think if somebody is crashing, time is very important so 30 seconds to wash your hands would change the patient's outcome, at that point it would be OK not to wash your hands. You could always wash your hands afterwards. If they are crashing, a virus is not their biggest problem at that point. Interview 4 
Residents described that they may avoid HH behaviours in scenarios where the risk appeared lower, in attempts to see many patients in a timely manner.

\begin{abstract}
Sometimes when you are busy or you know you just quickly want to do something, you're like-oh I'm just going to go into the very front of the room, I'm not going to contact this patient and I don't need to necessarily wash my hands, I'm just looking at him-you know something like that. Interview 6
\end{abstract}

At times residents felt that their own learning suffered if they did not keep up with the pace of the staff physician. If their staff physician did not perform $\mathrm{HH}$, the resident felt compelled to do the same to not miss any learning opportunities.

\section{Self-protection}

Residents described that they worried about getting sick themselves and this was a major motivator for adhering to $\mathrm{HH}$ regulations.

I always wash my hands before I eat and it is because I have this recurring image of spores being left on my hands and so I think it is really important because really, after having touched a patient and the wounds, and all these different things, it is important for us to be aware of the fact that we should probably clean our hands before we eat. Interview 6

The more likely you are to catch a severe illness, the more likely you are to do hand hygiene properly. Interview 15

While most residents were primarily concerned about their own health, a smaller number described secondary concern for others including their patients as well as their own family members.

Probably the biggest one [reason for doing $\mathrm{HH}$ ] that I didn't really mention before is the thought of dragging anything gross home to my children. Although I don't want to spread things in the hospital, also my own family is a more personal reason. Interview 21

[The main reason for doing $\mathrm{HH}$ is] probably getting sick yourself. Even if you do have good hand hygiene, you still catch colds, like I have right now. Personal protection is key, but as well you always want to protect your patients. Interview 22

I know that I touch a lot of things and we often have a fragile medical population, so I don't want to give them anything, and I don't want to get sick, which is a huge motivator. Interview 3

\section{Cues as part of HH habit}

Residents developed a habit of doing $\mathrm{HH}$ at certain times, for instance while taking a history, before a physical exam, and on the way out of a patient area. When the patient is on isolation the resident performs $\mathrm{HH}$ before going into the room, possibly implying that they might not do this in a non-isolated room.

My personal cue is I fit it ( $\mathrm{HH})$ into my natural history, or doing my examination, so I always do it before anything during my examination and then I do it on my way out as I am collecting my things from the room, so it is just a natural flow and then the only difference would be if the patient is on isolation, then you would be doing hand hygiene before entering the room. Interview 20

I think it is a habit. Most of the time I wash my hands, so I don't really remember when I don't wash my hands. Interview 11

If the patient coughs, then I'll be like-oh yeah and just wash my hands. Interview 22

If residents forget to engage in $\mathrm{HH}$ because of competing factors such as being distracted or caring for an acutely sick patient, they mention cues that serve as reminders.

If I miss one of the cues, I actually usually at least think about it when I am going to examine the patient, but that is one of the cues as well ...If I am walking into a room I may be distracted by a question or by a sick patient, and that is one of the times where I might get off my rhythm of coming in, washing my hands and going to see the patient. So anything that distracts me from my normal routine, which isn't that difficult for me, is probably going to pull me off of that. Interview 10

...I think sometimes you catch yourself. I was in PICU today and I had already washed my hands to examine part of a patient and then I was going to look at their abdominal wound and I was moving to do it and then I thought, 'Oh yeah' and then I went and washed my hands again and gloved. So, I suppose that is a near miss. Interview 19

In addition to these four major themes described above, residents self-reported their personal $\mathrm{HH}$ adherence at a range of $70-99 \%$. Most reported their $\mathrm{HH}$ to be greater than $90 \%$. While some residents described that they did not have a sense of other healthcare provider's $\mathrm{HH}$ adherence many perceived the hospital-wide adherence at $45-80 \%$ which was lower than the residents' compliance in general.

\section{DISCUSSION}

Four major themes were identified from interviews with paediatric residents regarding their attitudes and beliefs regarding $\mathrm{HH}$ including:

1. Importance of role models in promoting or decreasing $\mathrm{HH}$;

2. Balancing competing priorities;

3. Self-protection for residents primarily, followed by family and patient protection;

4. Cues as part of HH habit. 
The first theme identified was that of the importance of role modelling in $\mathrm{HH}$. This was further divided into three parts: (A) resident seeing him/herself as a positive role model for good $\mathrm{HH}$ for others including medical students; (B) the residents being influenced by more senior staff physicians regarding $\mathrm{HH}$ importance in both a positive or negative ways and (C) residents identifying that they could be positively influenced in a nonhierarchical manner, by seeing other healthcare team members wash their hands. The importance of staff physicians in role modelling has been previously described where $\mathrm{HH}$ upon entering patients' room on an internal medicine ward was $66 \%$ if the staff physician performed and $42 \%$ if they did not. ${ }^{13}$ This study also found that if the first person entering a patient room performed $\mathrm{HH}$, that this resulted in the increased likelihood of other team members (including residents and students) completing HH. ${ }^{13}$ This supports our theme of nonhierarchical promotion of HH. Another observational study supports residents' comments regarding the negative impact of decreased $\mathrm{HH}$ behaviour when a senior staff member (in this study either senior physician or nurse) did not engage in $\mathrm{HH}^{14}$ Based on our findings and the literature, interventions aimed at improving compliance among role models may be more successful initially, and then compliance may be sustained by less hierarchical cues of care providers seeing each other complete $\mathrm{HH}$ behaviours.

The second theme describes residents balancing competing priorities of $\mathrm{HH}$ with other factors including, but not limited to, busy schedules, high patient burden and medical emergencies. When there were other or multiple competing factors $\mathrm{HH}$ was more likely to suffer. A common sentiment among residents was that it was acceptable to neglect $\mathrm{HH}$ when an acute intervention was needed for a sick patient. Decreased HH compliance is associated with increased patient load as well as in the care of acutely ill patients, ${ }^{15}$ which was mentioned by several of our participants. Similar to our findings, another qualitative study described similar competing priorities, including interruptions and acutely sick patients related to decreased $\mathrm{HH}$ adherence. ${ }^{16}$ Higher-priority tasks, such as caring for sick patients may pre-empt or interrupt other tasks such as HH. The general phenomenon of interruption has been well described in the literature $^{17} 18$ as the cognitive shifts accompanying interruptions are potential causative agents for medical error. ${ }^{19}$ We posit that these cognitive shifts may similarly undermine intentions to comply with $\mathrm{HH}$ requirements.

The third theme identified through interviews was the importance of self-protection as a key motivator for paediatric residents to wash their hands. Previous studies have reported that self-protection drives healthcare providers to engage in $\mathrm{HH} .{ }^{16}{ }^{20}$ In our study, residents additionally reported that the desire to avoid transmitting infectious agents to their family members would drive $\mathrm{HH}$ behaviours, which is intuitive. In the light of recent public health epidemics such as avian influenza, the drive to protect one's own family may form an effective part of future interventions to improve $\mathrm{HH}$ compliance.

The last theme involved paediatric residents using cues to develop HH habit. These cues may help in 'nearmiss situations' when HH was almost forgotten but a cue provided a reminder to engage in $\mathrm{HH}$. Each task the resident engages in provides a cue for the next task within a larger routine. Habit as described in the interviews could be conceptualised as a series of cues to promote $\mathrm{HH}$ in this case. When the routine is disrupted, returning to the correct part of the routine so that no tasks are missed or duplicated requires the provider to remember where in the routine they were. ${ }^{18}$ Memory tends to be poor, particularly when the intervening task is cognitively demanding, or due to other factors. In our transcripts, contact events triggered efforts to recall - 'did I actually perform HH first?' Memory of an $\mathrm{HH}$ event can depend on the ease of retrieval of the last sensorimotor experience of hand washing, which leads to the recognition that an error was made. This error recognition process may also fail to varying degrees, accounting for some of the variability in the descriptions of adherence the residents observed. A recent study showed that reasons for performing $\mathrm{HH}$ can be thought of in the theory of planned behaviour as being internally motivated, but that reasons for not performing $\mathrm{HH}$ can be affected by the environment. ${ }^{21}$ The theory of planned behaviour is based on the intention of an individual to perform a behaviour (in this case: $\mathrm{HH}$ ). ${ }^{22}$ Based on our results, we posit that interruptions and their associated cognitive demands may lead to lower $\mathrm{HH}$ adherence, a hypothesis which may be investigated in the future. In our study, various cues helped to prevent 'near-misses' in $\mathrm{HH}$.

Residents' self-perception of their own $\mathrm{HH}$ was significantly higher than previously observed studies. Most published observational studies report that compliance of physicians and other healthcare professionals varies from $50 \%$ to $60 \%$ in different studies. ${ }^{13} 15$ In this study, residents self-report (and self-perceive) that their compliance with $\mathrm{HH}$ is $70-99 \%$. The term hand washing was mentioned in several interviews. Residents used hand washing to be synonymous with $\mathrm{HH}$ in our study. Most residents did not use soap and water to wash their hands but rather used alcohol-based hand sanitisers more frequently as a regular method of $\mathrm{HH}$. They would use soap and water if the situation warranted the same, for example, with a patient with Clostridium difficile diarrhoea. More recent studies show more promising $\mathrm{HH}$ compliance after a variety of interventions to promote $\mathrm{HH}^{10}{ }^{23}$ These interventions included education modules, ${ }^{23}$ encouraged shared accountability, ${ }^{23}$ leadership $^{10}$ and availability of hand sanitisers. ${ }^{10}$ In one study observed, HH compliance increased in both staff and resident physicians to more than $95 \%$ at the end of the study. ${ }^{23}$

The strengths of this study include our sample which originates in a large referral teaching hospital where 
$\mathrm{HH}$ compliance is quantitatively similar to that reported in comparable environments and averaging around 50\% in observed studies. ${ }^{6}$ Although most of these themes are supported in the literature by other observational and qualitative studies, this study focuses exclusively on paediatric resident physicians and identifies themes applicable to other healthcare providers including nurses and staff physicians. Our hospital is also unique because it is a 'hospital with in a hospital' in that the children's hospital is a part of the adult hospital. $\mathrm{HH}$ is promoted strongly at our institute, in particular in the children's hospital side and knowledge of resident physicians' attitudes towards $\mathrm{HH}$ is important in this promotion.

\section{CONCLUSION}

Staff physicians were viewed as being integral to initiating group $\mathrm{HH}$ events, but at times, the first person to enter the patient's room acted as a model for the rest of the group. In certain instances, such as a cardiac arrest, non-compliance with $\mathrm{HH}$ was viewed as acceptable. Residents engaged in $\mathrm{HH}$ to protect their own health. Residents have cues, which they integrate into their own $\mathrm{HH}$ habit. Incorporation of these findings in future strategies targeted at resident physicians during their training may improve compliance with $\mathrm{HH}$.

Contributors DD, SF, RH and TR conceived the idea of the study and were responsible for the design of the study. DD was responsible for data collection and initial qualitative analysis of transcribed interviews. SF, RH, TR and MB were also responsible for data analysis. All authors provided input into data analysis and theme development. The initial draft of the manuscript was prepared by DD and then circulated repeatedly among all authors for critical revision and interpretation of results.

Funding Funded by WCHRI: Women's and Children's Health Research Institute.

Competing interests None.

Ethics approval Human Research Ethics Board: University of Alberta.

Provenance and peer review Not commissioned; externally peer reviewed.

Data sharing statement No additional data are available.

\section{REFERENCES}

1. Boyce JM, Pittet D, Healthcare Infection Control Practices Advisory Committee, Society for Healthcare Epidemiology of America, Association for Professionals in Infection Control, Infectious Diseases Society of America. Hand Hygiene Task Force. Guideline for Hand Hygiene in Health-Care Settings: recommendations of the
Healthcare Infection Control Practices Advisory Committee and the HICPAC/SHEA/APIC/IDSA Hand Hygiene Task Force. Infect Control Hosp Epidemiol 2002;23(12 Suppl):S3-40.

2. Pittet D, Allegranzi B, Sax $\mathrm{H}$, et al. Evidence-based model for hand transmission during patient care and the role of improved practices. Lancet Infect Dis 2006;6:641-52.

3. Won SP, Chou HC, Hsieh WS, et al. Handwashing program for the prevention of nosocomial infections in a neonatal intensive care unit. Infect Control Hosp Epidemiol 2004;25:742-6.

4. Mathai E, Allegranzi B, Kilpatrick C, et al. Prevention and control of health care-associated infections through improved hand hygiene. Indian J Med Microbiol 2010;28:100-6.

5. Hall CB. Nosocomial viral respiratory infections: perennial weeds on paediatric wards. Am J Med 1981;70:670-6.

6. Hall CB, Douglas RG Jr. Modes of transmission of respiratory syncytial virus. J Pediatr 1981;99:100-3.

7. Ward DJ. Attitudes towards infection prevention and control: an interview study with nursing students and nurse mentors. BMJ Qual Saf 2012;21:301-6.

8. Ferguson $\mathrm{KJ}$, Waotzlom J, Beekmann SE, et al. Critical incidents of nonadherence with standard precautions guidelines among community hospital-based health care workers. J Gen Interm Med 2004;19:726-31.

9. Ji G, Yin H, Chen Y. Prevalence of and risk factors for non-compliance with glove utilization and hand hygiene among obstetrics and gynaecology workers in rural China. $J$ Hosp Infect 2005;59:235-41.

10. Jamal A, O'Grady G, Harnett E, et al. Improving hand hygiene in a paediatric hospital: a multimodal quality improvement approach. $B M J$ Qual Saf 2012;21:171-6.

11. Patton MQ. Qualitative research and evaluation methods. London: Sage Publications, Inc, 2001.

12. Guba EG, Lincoln YS. Competing paradigms in qualitative research. In: Denzin NK, Lincoln YS, eds. Handbook of qualitative research. Thousand Oaks, CA: Safe Publications, Inc, 1984:105-17.

13. Haessler S, Bhagavan A, Kleppel R, et al. Getting doctors to clean their hands: lead the followers. BMJ Qual Saf 2012;21:499-502.

14. Lankford MG, Zembower TR, Trick WE, et al. Influence of role models and hospital design on hand hygiene of health care workers. Emerg Infectious Dis 2003;9:217-23.

15. Pittet D, Mourouga P, Perneger TV, et al. Compliance with handwashing in a teaching hospital. Ann Intern Med 1999;130: 12-30.

16. Jang JH, Wu S, Kirzner D, et al. Focus group study of hand hygiene practice among healthcare workers in a teaching hospital in Toronto, Canada. Infect Control Hosp Epidemiol 2010;31:144-50.

17. Chisholm CD, Collison EK, Nelson DR, et al. Emergency department workplace interruptions: are emergency physicians ' interrupt-driven' and 'multitasking'? Acad Emerg Med 2000;7:1239-43.

18. Westbrook JI, Coiera E, Dunsmuir WT, et al. The impact of interruptions on clinical task completion. Qual Saf Health Care 2010;19:284-9.

19. Rivera-Rodriguez AJ, Karsh B-T. Interruptions and distractions in healthcare: review and reappraisal. Qual Saf Health Care 2010;19:304-12.

20. Borg MA, Benbachir M, Cookson BD, et al. Self-protection as a driver for hand hygiene among healthcare workers. Infect Control Hosp Epidemiol 2009;30:578-80.

21. McLaughlin AC, Walsh F. Self-reported reasons for hand hygiene in 3 groups of health care workers. Am J Infect Control 2012;40:653-8.

22. Ajzen I. From intentions to actions: a theory of planned behavior. In: Kuhl J, Bekmaan J, eds. Action control: from cognition to behavior. New York: Springer-Verlag, 1985:11-39.

23. White CM, Statile AM, Conway PH, et al. Utilizing improvement science methods to improve physician compliance with proper hand hygiene. Paediatrics 2012;129:e1042-50. 